Check for updates

Cite this: RSC Adv., 2017, 7, 23407

Received 9th February 2017

Accepted 21st April 2017

DOI: $10.1039 / \mathrm{c} 7 \mathrm{ra01636k}$

rsc.li/rsc-advances

\section{Effective surface attachment of Ag nanoparticles on fibers using glycidyltrimethylammonium chloride and improvement of antimicrobial properties $\dagger$}

\author{
Chankyu Kang, (D) ta Sam Soo Kim, t $^{\mathrm{b}}$ Dajeong Ahn, ${ }^{\mathrm{b}}$ Soo Jung Kim ${ }^{\mathrm{b}}$ \\ and Jaewoong Lee (D)*b
}

Functional poly( $m$-phenylene isophthalamide), $m$-aramid (known as Nomex®) fibers with antimicrobial properties were prepared by applying quaternary ammonium salts (such as glycidyltrimethylammonium chloride (GTAC)) in combination with silver nanoparticles (AgNPs). The fibers treated by this simple process exhibited enhanced antimicrobial activity. In the coating process, the $m$-aramid fibers were immersed in a GTAC solution and reacted via the pad-dry-cure process. The GTAC-treated $m$-aramid fibers were then reacted with an $\mathrm{Ag}$ colloid solution at $40{ }^{\circ} \mathrm{C}$ for $90 \mathrm{~min}$ to prepare GTAC/AgNP-treated $m$-aramid fibers. Scanning electron microscopy was used to confirm the surface morphology of the $m$ aramid fibers treated with GTAC and AgNPs. Changes in the chemical composition before and after GTAC and AgNP treatment were analyzed by scanning electron microscopy with energy-dispersive X-ray spectroscopy. The tensile strength of the GTAC/AgNP-treated $m$-aramid fibers declined by about $3.5 \%$ compared to that of untreated $m$-aramid fibers. Durability of the AgNPs on the $m$-aramid fibers treated with GTAC/AgNPs was demonstrated through a washing-fastness test, indicating $76 \%$ retention after five washing cycles. The antimicrobial activity analysis showed that the synergistic antimicrobial properties of the GTAC/AgNP-treated $m$-aramid fibers resulted in efficacy against $P$. aeruginosa.

\section{Introduction}

With the recent emergence of technical textiles, the focus in the textile industry is rapidly shifting from regular textile fibers to technical textile fibers. Poly ( $m$-phenylene isophthalamide), $m$ aramid fibers (known as Nomex®) exhibit excellent heat resistance, chemical resistance, abrasion resistance, electrical insulation, and hygroscopic properties, and are widely industrially applicable. ${ }^{1-5}$ In recent years, $m$-aramid fibers have been successfully commercially applied in safety work clothes, aircraft seats, arctic clothes, and aircraft blankets. ${ }^{6,7}$ However, $m$-aramid fibers do not inherently possess antimicrobial properties. For example, in the case of firefighter uniforms, microorganisms may proliferate due to exposure to moisture. ${ }^{8}$ Therefore, when the high-performance characteristics of $\mathrm{m}$ aramid are combined with strong antimicrobial properties and

${ }^{a}$ Ministry of Employment and Labor, Major Industrial Accident Prevention Center, 34 Yeosusandallo, Yeosu-si 59631, Republic of Korea

${ }^{b}$ Department of Textile Engineering and Technology, Yeungnam University, 280 Daehak-Ro, Gyeongsan, Gyeongbuk 38541, Republic of Korea. E-mail: jaewlee@yu. ac.kr; Fax: +82 53810 4685; Tel: +82 538102786

$\dagger$ Electronic supplementary information (ESI) available. See DOI: 10.1039/c7ra01636k

\$ Chan Kyu Kang and Sam Soo Kim contributed equally to this work. durability that can be maintained even with repeated use, it is feasible for these fibers to become a material of choice.

Even though not all microorganisms are dangerous, some microorganisms (i.e., virus, fungi, and bacteria) can cause damage to fiber materials and induce human infections., ${ }^{9,10}$ In order to minimize the damage caused by these microorganisms, various methods such as spraying, ${ }^{11}$ padding, ${ }^{12}$ dipping, ${ }^{13}$ and nanocoating with inorganic antimicrobial materials ${ }^{14}$ have been developed for imparting antimicrobial properties to the fibers. However, these methods have the following disadvantages: ${ }^{15,16}$ (i) they induce deterioration of the physical properties of the fibers due to the application of external stimulation such as heat to induce antimicrobial activity, (ii) the antimicrobial activity is non-continuous, and (iii) contamination by microorganisms is possible.

Inorganic antimicrobial materials that are harmless to the human body and possess excellent stability (i.e., are non-volatile and do not readily decompose) have been widely popularized., ${ }^{4,17}$ Among the known inorganic antimicrobial materials, silver nanoparticles (AgNPs) have been the focus of continuous interest and research due to their desirable optical and conductive properties. ${ }^{19-21}$ For example, optical characteristics are used for biosensors and numerous assays, and the conductive properties are used for conductive inks and integrated into composites that enhance thermal and electrical 
conductivity. ${ }^{22-25}$ In addition to these applications, AgNPs, which have dramatically increased surface area compared to bulk silver particles, exhibit excellent antimicrobial properties at very low loading, and exhibit excellent resistance and sterilizing power against hundreds of harmful microorganisms. ${ }^{26-30}$ This feature allows AgNPs to be applied to a variety of fiberforming polymers such as polypropylene, polyethylene and polystyrene as well as natural fibre. ${ }^{31-34}$ Even though the detailed mechanism has not been fully elucidated, the particle size, shape, crystallinity, material surrounding the particles, $\mathrm{pH}$ of the solution, ionic strengths, types of ions, and ligand are significant factors influencing the antimicrobial activity of AgNPs. ${ }^{35,36}$ It is generally known that the smaller the size of the AgNPs, the greater is the number of atoms on the surface that can come into contact with bacteria, and the greater amount of silver ions can be released. ${ }^{37}$ Microbial inactivation using AgNPs has two main effects. Firstly, direct contact with AgNPs destroys and inactivates the cell membrane. During this process, the AgNPs permeate microbial cells and induce damage to sulfurand phosphorous-containing compounds. ${ }^{38}$ Secondly, silver ions released from the AgNPs inactivate microorganisms..$^{27,39,40}$ Disk-diffusion, well diffusion, broth or agar dilution, flow cytofluorometric and bioluminescent methods are used to measure antimicrobial activity using AgNPs. ${ }^{41}$

Synthetic organic compounds such as quaternary ammonium compounds (QACs) are considered as effective antimicrobial agents and are primarily used to inhibit bacterial growth and secondarily to increase the durability of fiber materials. ${ }^{42}$ QACs are mainly composed of four hydrocarbon groups bonded to nitrogen atoms. The cationic (+) group of the QAC is adsorbed through the anionic $(-)$ portion of the microbial cell surface by electrostatic attraction, thereby destroying the cell structure and resulting in an increase of the antimicrobial activity. ${ }^{\mathbf{4 3 4 4}}$ As a method of applying AgNPs to several types of fibers, 3-mercaptopropyltrimethoxysilane, ${ }^{45}$ chitosan, ${ }^{46}$ and polycarboxylic acids ${ }^{47}$ have been used as cross-linking agents that promote strong bonding and inhibit the growth of bacteria.

The purpose of this study is to introduce a simple method for imparting effective antimicrobial properties to fibers. GTAC is one of quaternary ammonium salts which are a kind of antimicrobial agent and AgNPs is another kind of antimicrobial agent. The combination of GTAC/AgNPs on the fiber surface analyzed synergistic antimicrobial effects. To the best of our knowledge, it is the first demonstration of effective AgNPs attachment on $m$-aramid fibers using glycidyltrimethylammonium chloride (GTAC), which is containing an epoxy ring. In addition, this study introduced AgNPs directly attached into $m$-aramid fibers without the aid of cross-linking agent. Surface properties, physical and thermal properties, durability and antimicrobial efficacy of $m$-aramid fibers by direct reaction of the GTAC/AgNPs were investigated. To verify the properties of fibers, several analysis methods (i.e. TEM, SEM-EDS, washing-fastness test, tensile strength measurement, thermogravimetric analysis, antimicrobial test) were used. Throughout these studies, we expect that AgNPs can be conveniently deposited on $m$-aramid fibers and commercially available $m$-aramid fibers with excellent antibacterial efficacy and durability of AgNPs.

\section{Experimental section}

\subsection{Materials}

GTAC (90\%) was purchased from Sigma Aldrich (St. Louis, MO, USA), and silver colloid (Nanomix-silver 30000 ppm) was obtained from Miji Nanotech (Seoul, Korea). Both products were used without further purification. The $m$-aramid fibers obtained from Testfabrics Inc. (West Pittston, PA, ASTM Nomex Defender 750 YELLOW) were refined and desized. To purify the $m$-aramid fibers, the samples were washed twice with distilled water at $60{ }^{\circ} \mathrm{C}$ and washed twice with ethanol and acetone, respectively. The treated samples were dried under ambient conditions for 24 hours.

\subsection{Quaternary ammonium salt-treated $m$-aramid fibers}

In order to treat the $m$-aramid fibers with quaternary ammonium salts, solutions of GTAC (a quaternary ammonium salt) were prepared at respective concentrations of 2, 4, 6, 8, 10, 20, 30 , and $40 \mathrm{wt} \%$ using distilled water as a solvent. The pad-drycure process was used for preparation of the sample, and the specific method was as follows. The $m$-aramid fibers were immersed in $50 \mathrm{~mL}$ of GTAC solution prepared at each concentration for $30 \mathrm{~min}$ at ambient temperature without shaking. The treated samples were kept at $80{ }^{\circ} \mathrm{C}$ for $30 \mathrm{~min}$ for drying, followed by curing at $190{ }^{\circ} \mathrm{C}$ for $15 \mathrm{~min}$. Finally, the treated samples were rinsed with distilled water to remove unreacted GTAC and dried under ambient conditions.

\subsection{Silver nanoparticle-coated $m$-aramid fibers}

The untreated and GTAC-treated $m$-aramid fibers were immersed in $15 \mathrm{~mL}$ of silver colloid and shaken in a water bath at $40{ }^{\circ} \mathrm{C}$ for $90 \mathrm{~min}$ at $110 \mathrm{rpm}$. After the coating process, the fibers were washed twice with distilled water and dried naturally.

\subsection{Transmission electron microscopy (TEM)}

A transmission electron microscope (H-7600, Hitachi, Tokyo, Japan) was used to observe the size and distribution of the AgNPs in the colloid. The acceleration voltage was $120 \mathrm{kV}$ with a resolution of $0.32 \mathrm{~nm}$.

\subsection{Scanning electron microscope-energy dispersive X-ray spectroscopy (SEM-EDS) analysis}

Analysis of the morphological changes of the antimicrobial $\mathrm{m}$ aramid fibers and elemental analysis of the chemical composition of the sample surface were performed by using SEM-EDS (S-4100, Hitachi, Tokyo, Japan). The surface of the fibers was observed using SEM-EDS with an accelerating voltage of $15 \mathrm{kV}$. The samples were coated with platinum by sputtering before the examination. In order to compare the surface composition of the pure $m$-aramid fibers with that of the antimicrobial treated $m$-aramid fibers, each surface was observed at $\times 3 \mathrm{k}$ magnification using energy dispersive component analysis. 


\subsection{Washing-fastness test}

For the washing-fastness test, the KS test method (K IOS 105C01: 2007) was used to determine the durability of the AgNPs bound to the $m$-aramid fibers. This method involved the use of a cylinder made of glass or stainless steel with a diameter of (75 $\pm 5) \mathrm{mm}$, height of $(125 \pm 10) \mathrm{mm}$, and volume of $(550 \pm 50)$ $\mathrm{mL}$. An aqueous detergent solution $\left(5 \mathrm{~g} \mathrm{~L}^{-1}\right)$ was prepared by using AATCC 2003 standard reference liquid detergent without an optical brightener (Test fabrics Korea, Inc., Ansan, Korea). The prepared solution was placed in a cylinder at a solution $/ \mathrm{m}$ aramid fiber ratio of $50: 1$ and washed at a temperature of $40 \pm$ $2{ }^{\circ} \mathrm{C}$ for $30 \mathrm{~min}$ at a rotation speed of $40 \pm 2 \mathrm{~min}^{-1}$ for $30 \mathrm{~min}$. After the washing process, the test specimens were washed with cold water for $2 \mathrm{~min}$ and dried at room temperature.

\subsection{Tensile strength}

The mechanical properties of the $m$-aramid fibers treated with GTAC and AgNPs were evaluated using the ASTM D 7269 test method. Test specimens with a width of $100 \pm 2 \mathrm{~mm}$ and a length of $150 \mathrm{~mm}$ were prepared in accordance with the test method before analysis. The tensile testing instrument (Model 3345, Instron Corp., Canton, MA, USA) was set at a holding distance of $75 \pm 1 \mathrm{~mm}$ and a stretching speed of $300 \pm 10 \mathrm{~mm}$ $\min ^{-1}$. The alignment line on the test specimen was grasped to touch the side surface of the front jaw, with a width of $25 \mathrm{~mm}$ at the top and bottom; the specimen was then placed in a straight line and the process was repeated at least five times.

\subsection{Thermogravimetric analysis (TGA)}

The thermal analysis data, including the TGA weight loss curve, were acquired using a TG-DTA (SDT Q600, TA Instruments, Delaware, USA) instrument to analyze the thermal properties of the untreated $m$-aramid fibers, GTAC, and the Ag colloid-treated $m$ aramid fibers. The tests were performed under $\mathrm{N}_{2}$ atmosphere and the temperature was ramped to $600{ }^{\circ} \mathrm{C}$ at a heating rate of $10{ }^{\circ} \mathrm{C}$ $\min ^{-1}$.

\subsection{Antimicrobial test}

The strains used for the antimicrobial test were Escherichia coli O157:H7 (ATCC 43895) and Pseudomonas aeruginosa PAO1 (ATCC 15692) as Gram-negative bacteria and Staphylococcus aureus MRSA (ATCC BAA-1707) as Gram-positive bacteria. Luria-Bertani (LB; ATCC bioscience, NJ, USA), Nutrient Broth (NB; ATCC Medium, Sigma-Aldrich, USA), and Agar (Difco) were used for the growth of each strain. The cryo-preserved bacterium was placed into the liquid medium and was activated in an incubator for 24 hours at $37{ }^{\circ} \mathrm{C}$. A $1 \mathrm{~mL}$ aliquot of the activated liquid medium was inoculated into a sample with dimensions of $1 \times 1 \mathrm{~cm}^{2}$. After 24 hours, the treated sample was added to $0.85 \%$ of physiological saline solution and vortexed, and the diluted solution (controlled by a decimal system) was used for the experiment. The antimicrobial activity was measured using the Disc Diffusion Method (Halo test) ${ }^{48}$ and the AATCC Test Method 100 (Contact Assay in Liquid).

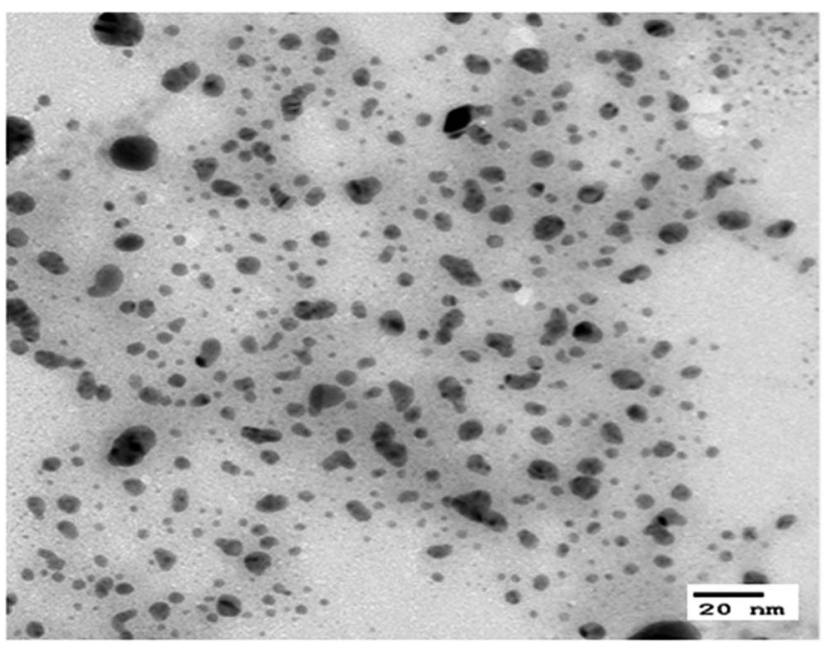

Fig. 1 TEM image of AgNPs in the silver colloid solution.

\section{Results and discussion}

\subsection{Surface analysis of GTAC and AgNP-treated samples}

The distribution and size of the dispersed AgNPs in the silver colloid solution used for attaching the AgNPs to the surface of the $m$-aramid fibers were measured by using TEM and the images are presented in Fig. 1. The measured size of the AgNPs was generally found to be less than $20 \mathrm{~nm}$, and the mean size of the AgNPs was observed to be about $11.49 \pm 2 \mathrm{~nm}$. The stabilized AgNPs were formed in solution, and most of the bare AgNPs were involved in the reaction. It is known that in water, aggregation of the AgNPs is hindered because the asymmetrical charge distribution on the metal nanoparticle surfaces can be minimized by dissociated water. ${ }^{\mathbf{4 9 , 5 0}}$ The proposed experimental plan for inducing effective bonding of the AgNPs on the $\mathrm{m}$ aramid fibers is illustrated in Scheme 1.

In order to compare the surface properties of the pure $m$ aramid fibers with those of the $m$-aramid fibers treated with antimicrobial materials, the morphology of each surface, as shown in Fig. 2, was observed at a magnification of $\times 3000$ using a SEM instrument. The data indicated that the untreated $\mathrm{m}$ aramid fibers had a relatively smooth surface (as in Fig. 2a). As
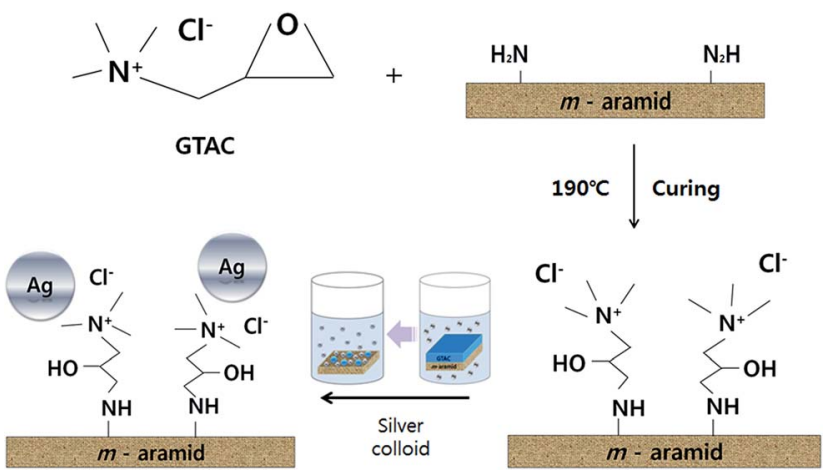

Scheme 1 Proposed scheme for preparation of GTAC/AgNP-treated $m$-aramid. 


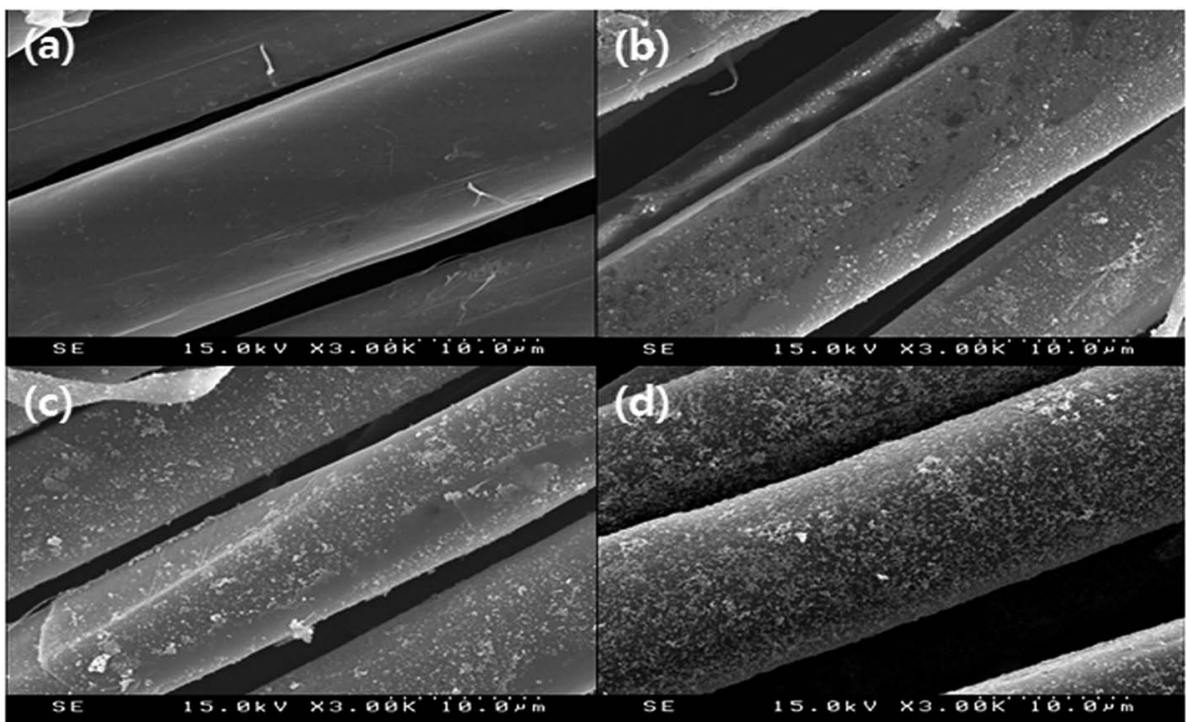

Fig. 2 SEM micrograph of (a) native $m$-aramid and (b) $m$-aramid treated with 10 wt\% GTAC/2500 ppm AgNPs, (c) $m$-aramid treated with 10 wt\% GTAC/10 000 ppm AgNPs, (d) m-aramid treated with 10 wt\% GTAC/30 000 ppm AgNPs.

the concentration of the AgNPs increased, the surface of the treated $m$-aramid fibers gradually became rough. In particular, the surface of the $m$-aramid fibers treated with $10 \mathrm{wt} \% \mathrm{GTAC} /$ AgNPs at a concentration of $30000 \mathrm{ppm}$ showed significant changes (as in Fig. 2d). Fig. 3 shows the SEM-EDS data for comparative analysis of the surface elemental composition of the untreated $m$-aramid fibers and the $m$-aramid fibers treated with GTAC and AgNPs. The profile of the $m$-aramid fibers
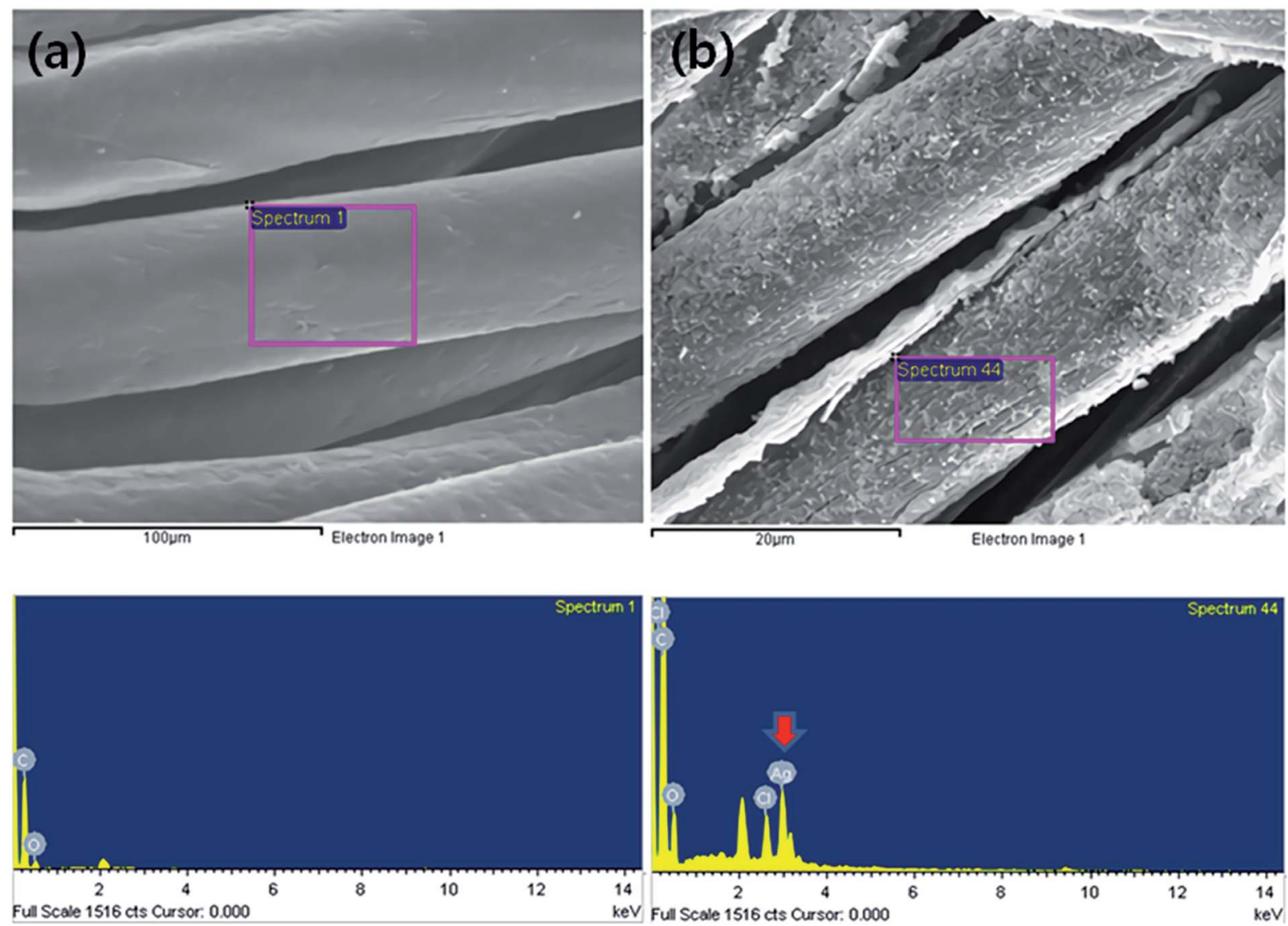

Fig. 3 SEM-EDS profile of (a) native $m$-aramid and (b) $m$-aramid treated with $10 w t \%$ GTAC/30 000 ppm AgNPs. 

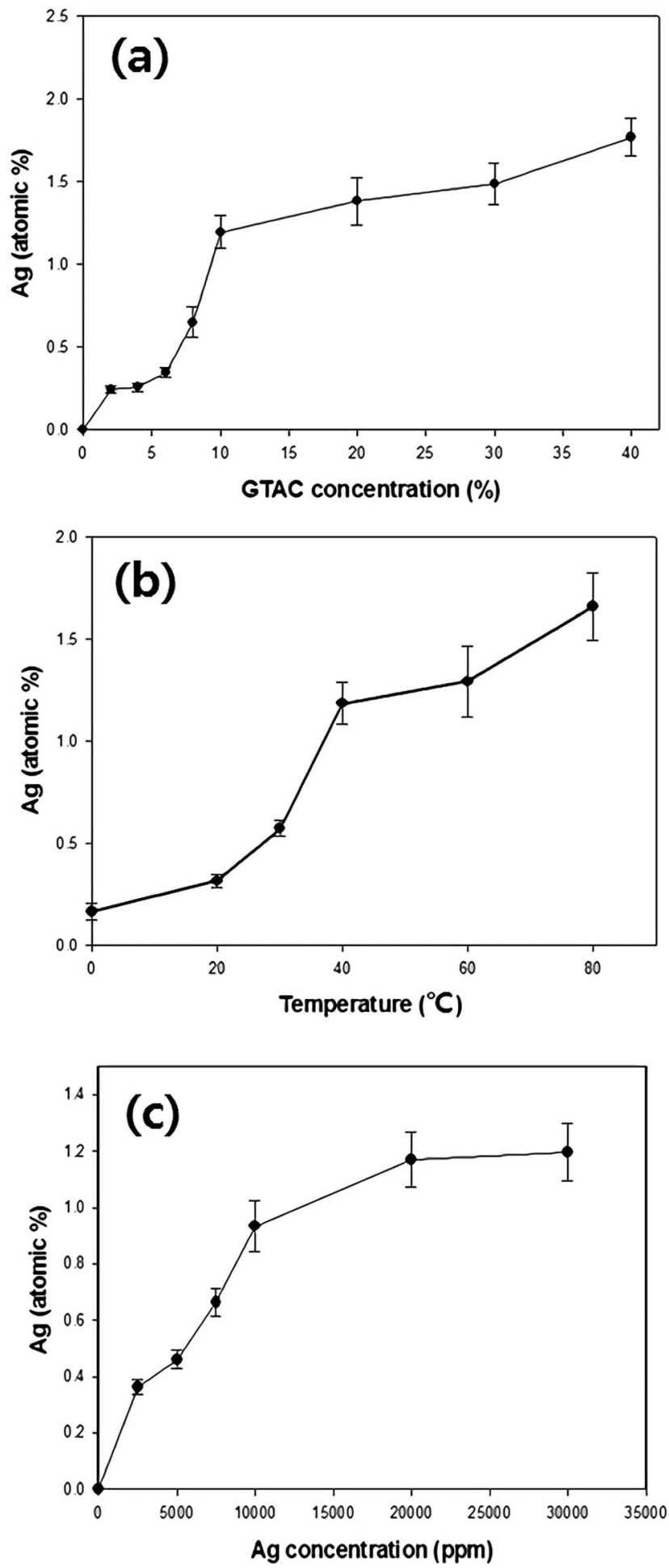

Fig. 4 Ag loading (atomic\%) on GTAC/AgNP-treated $m$-aramid fibers with variation of the (a) GTAC concentration, (b) temperature, and (c) Ag colloid concentration.

treated with GTAC/AgNPs (as in Fig. $3 \mathrm{~b}$ ) showed a peak of Ag from the AgNPs and a $\mathrm{Cl}$ peak from GTAC, as compared with that of the untreated $m$-aramid fibers. Based on the results in Fig. 2 and 3, it was found that GTAC and the AgNPs were successfully bound to the $m$-aramid fibers, and with increasing
Table 1 Tensile strength of native and GTAC/AgNP-treated $m$-aramid

\begin{tabular}{llll}
\hline $\begin{array}{l}\text { Control } \\
\text { treated with } \\
\text { distilled water })\end{array}$ & $\begin{array}{l}\text { Treated with } \\
\text { GTAC }\end{array}$ & $\begin{array}{l}\text { Treated with } \%) \\
\text { GTAC } \\
(10 \mathrm{wt} \%)\end{array}$ & $\begin{array}{l}\text { Treated with } \\
\text { GTAC }(10 \mathrm{wt} \%) / \\
\text { AgNPs }(30000 \mathrm{ppm})\end{array}$ \\
\hline $27.12 \pm 0.42$ & $27.03 \pm 0.98$ & $26.62 \pm 0.61$ & $26.17 \pm 0.61$
\end{tabular}

GTAC concentration, the AgNPs reacted with GTAC on the $m$ aramid fibers, leading to a rough surface. These results confirmed that the peak intensity was intensified by the increase of $\mathrm{OH}$-groups at $3200 \mathrm{~cm}^{-1}$ due to epoxy ring opening at the surface of $m$-aramid fibers by GTAC (ESI $1 \dagger$ ).

Results of analysis of the reactivity of Ag (atomic\%) towards the $m$-aramid fibers as a function of various factors are shown in Fig. 4. The change in the loading of $\mathrm{Ag}$ (atomic\%) on the $\mathrm{m}$ aramid fibers as a function of the GTAC concentration is presented in Fig. 4a. Increasing the concentration of GTAC enhanced the amount of $\mathrm{Ag}$ (atomic\%) attached to the $\mathrm{m}$ aramid fibers. Specifically, the loading of $\mathrm{Ag}$ (atomic\%) increased sharply with the addition of up to $10 \mathrm{wt} \%$ GTAC, beyond which the increase in the loading of $\mathrm{Ag}$ (atomic\%) was relatively slow. This is presumably because the number of amine groups $\left(-\mathrm{NH}_{2}\right)$ on the $m$-aramid fiber that can chemically react with GTAC was limited; thus, despite the increase in the concentration of GTAC, only a certain concentration of GTAC could react with the amine groups of the $m$-aramid fibers. Fig. $4 \mathrm{~b}$ shows the loading of $\mathrm{Ag}$ (atomic\%) on the $m$-aramid fibers with variation of the treatment temperature. The loading of AgNPs increased almost linearly with increasing treatment temperature. This is because at higher temperature, movement of the AgNPs in the treatment solution is enhanced, leading to greater migration to GTAC attached to the $m$-aramid fibers. The variation of the loading of $\mathrm{Ag}$ (atomic\%) on the $\mathrm{m}$-aramid fibers as a function of the concentration of the $\mathrm{Ag}$ colloid solution, is shown in Fig. 4c. As the concentration of the Ag colloid solution increased up to $30000 \mathrm{ppm}$, the loading of AgNPs on the $\mathrm{m}$ aramid fibers increased. The loading of $\mathrm{Ag}$ (atomic\%) increased markedly in the concentration range of the $\mathrm{Ag}$ colloid solution of 0 to $10000 \mathrm{ppm}$; however, the loading increased to a lesser extent in the range of 10000 to $30000 \mathrm{ppm}$. It is presumed that the GTAC-treated $m$-aramid fibers have a certain number of GTAC functional groups that are used to bind the AgNPs; thus, it is easy to bind AgNPs up to a certain concentration of the Ag. colloid solution. However, the binding between GTAC and AgNPs approaches saturation at higher Ag colloid concentrations, thereby limiting attachment of additional AgNPs.

\subsection{Physical and thermal properties}

Table 1 shows the variation of the tensile strength of the $m$ aramid fibers before and after treatment with the GTAC/AgNPs. The tensile strength of the GTAC/AgNP-treated $m$-aramid fibers declined slightly (by about 3.5\%) compared to that of the untreated $m$-aramid fibers. This means that there was no 


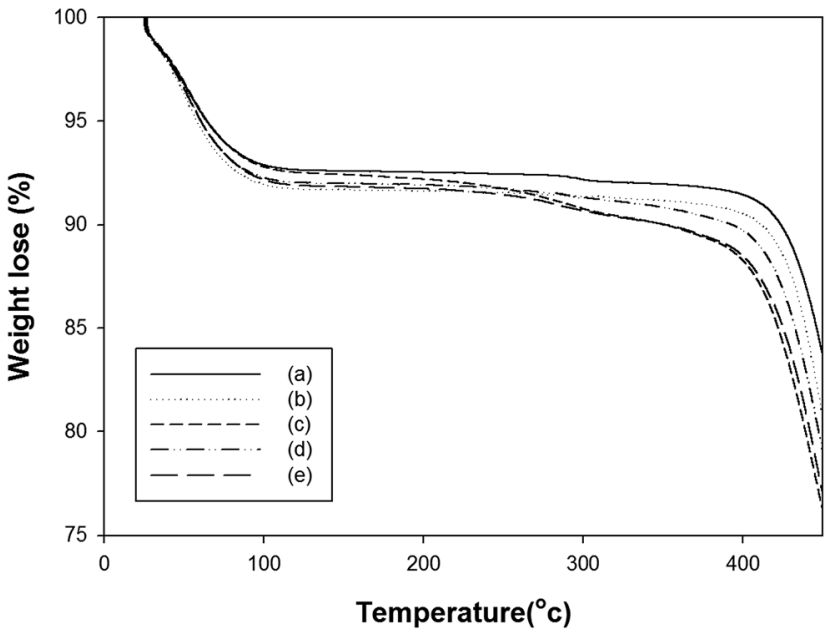

Fig. 5 TGA pattern of (a) native $m$-aramid fibers and (b) $m$-aramid fibers treated with $10 \mathrm{wt} \%$ of GTAC, (c) $m$-aramid fibers treated with 10 wt\% of GTAC/2500 ppm of AgNPs, (d) $m$-aramid fibers treated with 10 wt\% of GTAC/10 000 ppm of AgNPs, (e) $m$-aramid fibers treated with 10 wt $\%$ of GTAC/30 000 ppm of AgNPs.

Table 2 Washing-fastness of GTAC/AgNP-treated $m$-aramid

\begin{tabular}{lll}
\hline Washing (cycles) & $\mathrm{Ag}$ (atomic\%) & Retention (\%) \\
\hline 0 & 1.19 & 100 \\
1 & 1.13 & 94.96 \\
2 & 1.07 & 89.92 \\
3 & 0.99 & 83.19 \\
4 & 0.91 & 76.47 \\
5 & 0.90 & 75.63
\end{tabular}

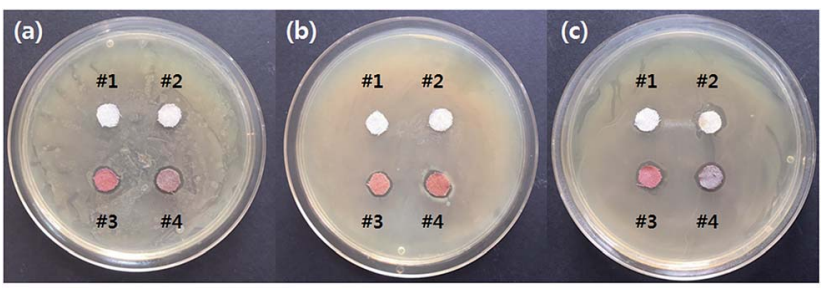

E. coli

S. aureus

P. aeruginosa

Fig. 6 Antimicrobial activity of \#1 native $m$-aramid fibers, \#2 $m$ aramid fibers treated with GTAC, \#3 $m$-aramid fibers treated with AgNPs, and \#4 $m$-aramid fibers treated with GTAC/AgNPs. significant change of the dynamic properties of the $m$-aramid fiber even after GTAC/AgNP treatment.

Thermograms of the untreated $m$-aramid fibers, GTAC-treated $m$-aramid fibers, and $m$-aramid fibers treated with GTAC and different concentrations of the Ag colloid are presented in Fig. 5 . The initial degradation temperature (IDT) of the $m$-aramid fibers was $430^{\circ} \mathrm{C}$ (as in Fig. 5a). On the other hand, the IDT of the GTACtreated $m$-aramid fibers decreased slightly $\left(423{ }^{\circ} \mathrm{C}\right)$. The thermal stability of the GTAC/AgNP-treated $m$-aramid fibers decreased compared to those of the $m$-aramid fibers treated with GTAC and also decreased as the concentration of AgNPs increased. The decrease in the IDT is thought to be due to the fact that GTAC, which has relatively poor thermal stability as compared with $m$ aramid fiber, adheres to the $m$-aramid fibers. It is also postulated that the metal particles (AgNPs) do not help to improve the thermal stability of the $m$-aramid fibers.

\subsection{Durability and antimicrobial efficacy}

Durability of the AgNPs attached to the GTAC-treated $m$-aramid fibers was evaluated after the washing test (Table 2). The durability is important because this property is required to maintain the superior functionality of the functional fibers. In the case of the $m$-aramid fibers used in this study, (as shown in Table 2) a retention ratio of about $76 \% \mathrm{Ag}$ was obtained after five wash cycles. In previous studies, AgNPs attached to 3-MPTMS-treated cotton fibers exhibited $66 \%$ retention after five wash cycles, while $m$-aramid fibers treated with GTAC and AgNPs exhibited improved retention despite the use of different materials. ${ }^{37}$

As mentioned, the purpose of this study is to fabricate synthetic fibers with enhanced antimicrobial efficacy by simultaneously applying GTAC, a quaternary ammonium salt, and AgNPs, another antimicrobial agent. The antimicrobial properties of the untreated and treated $m$-aramid fibers were verified as shown in Fig. 6 and Table 3. Fig. 6 shows the results of the Halo test, a qualitative method used to visually observe the generation of the zone of inhibition around a sample by placing the sample on a microorganism-coated medium. In the case of the samples treated with AgNPs alone (\#3) and the combination of GTAC/ AgNPs (\#4), inhibition bands appeared for the three types of bacteria. To further confirm the antimicrobial performance, a quantitative test method, the count reduction method, was applied (Table 3). In the case of E. coli and S. aureus, the bacteria were simultaneously inactivated in the samples treated with GTAC

Table 3 Antimicrobial test results for native and treated $m$-aramid

\begin{tabular}{|c|c|c|c|}
\hline \multirow[b]{2}{*}{ Samples } & \multicolumn{3}{|c|}{ Bacterial no. (cfu per sample) } \\
\hline & E. coli ${ }^{a}$ & S. aureus $^{b}$ & P. aeruginosa ${ }^{c}$ \\
\hline Native $m$-aramid (\#1) & $6.00 \times 10^{5}$ & $7.15 \times 10^{5}$ & $7.8 \times 104$ \\
\hline$m$-Aramid treated with GTAC $(\# 2)^{d}$ & 0 & $1.44 \times 10^{3}$ & $1.70 \times 103$ \\
\hline$m$-Aramid treated with AgNPs $(\# 3)^{e}$ & 0 & 0 & $1.40 \times 102$ \\
\hline$m$-Aramid treated with GTAC/AgNPs $(\# 4)^{f}$ & 0 & 0 & 0 \\
\hline$m$-Aramid treated with GTAC/AgNPs after 5 washing cycles $^{g}$ & 0 & 0 & 0 \\
\hline
\end{tabular}

${ }^{a}$ Total bacteria: $4.8 \times 10^{6} \mathrm{cfu}$ per sample. ${ }^{b}$ Total bacteria: $7.3 \times 10^{6} \mathrm{cfu}$ per sample. ${ }^{c}$ Total bacteria: $9.6 \times 10^{6}$ cfu per sample. ${ }^{d}$ Sample treated with $10 \mathrm{wt} \%$ GTAC. ${ }^{e}$ Sample treated with 30000 ppm AgNPs. ${ }^{f}$ Sample treated with $10 \mathrm{wt} \% \mathrm{GTAC} / 30000 \mathrm{ppm}$ AgNPs (Ag (atomic\%), 1.19$) .{ }^{g} \mathrm{Ag}$ (atomic\%), 0.90 . 
(\#2), AgNPs (\#3), and GTAC/AgNPs (\#4). On the other hand, $P$. aeruginosa, classified as a relatively strong strain, was not inactivated with GTAC only (\#2) or the AgNPs (\#3), but was inactivated by the $m$-aramid fibers treated with GTAC/AgNPs (\#4). This means that the $m$-aramid fibers treated with GTAC/AgNPs exhibited increased antimicrobial activity against $P$. aeruginosa relative to the $m$-aramid fibers treated with GTAC or AgNPs alone.

\section{Conclusion}

GTAC and AgNPs were simultaneously and successfully attached to the surface of $m$-aramid fibers. The washing-fastness test proved that the durability of the AgNPs on the $m$-aramid fibers was relatively high even after five repeated washes. The GTAC- and GTAC/AgNP-treated $m$-aramid fibers exhibited slightly (just 3.5\%) reduced tensile strength compared to the untreated $m$-aramid fibers. The GTAC/AgNP-treated $m$-aramid fibers exhibited enhanced antimicrobial properties compared to the $m$-aramid fibers treated with GTAC alone or with AgNPs alone. It can be concluded that $m$-aramid fibers treated with GTAC/AgNPs exhibit increased antimicrobial activity without changes of the physical properties of the fibers and can be used as a multifunctional fiber. Furthermore, $m$-aramid fibers can be widely applied in industrial textile fields, including medical textiles; thus, the treated fibers presented herein are considered promising.

\section{Acknowledgements}

This study was supported by a Yeungnam University Research Grant in 2015.

\section{References}

1 J. K. Fink, High performance polymers, William Andrew Inc., Norwich, U.K., 2008, pp. 423-448.

2 J. M. García, F. C. García and F. Serna, Prog. Polym. Sci., 2010, 35, 623-686.

3 H. G. Chae and S. Kumar, J. Appl. Polym. Sci., 2006, 100, 791802.

4 B. Wang, Y. Duan and J. Zhang, Mater. Des., 2016, 103, 330338.

5 N. N. Baeva, E. A. Manyukov, S. F. Sadova, Y. Konovalova and G. S. Negodyaeva, Fibre Chem., 2007, 39, 205-209.

6 E. M. Kim and J. Choi, Fibre Chem., 2011, 12, 484-490.

7 E. A. Manyukov, S. F. Sadova, A. S. Keck'yan, N. P. Puzikova and N. N. Baeva, Theor. Found. Chem. Eng., 2007, 41, 698-702.

8 M. Zanoaga and F. Tanasa, Chem. J. Mold., 2014, 9, 14-32.

9 M. L. Cohen, Science, 1992, 257, 1050-1055.

10 Y. Sun and G. Sun, Appl. Polym. Sci., 2001, 81, 1517-1525.

11 S. Snega, K. Ravichandran, N. J. Begum and K. Thirumurugan, J. Mater. Sci.: Mater. Electron., 2013, 23, 135-141.

12 W. Ye, M. F. Leung, J. Xin and P. Li, Polymer, 2005, 46, 1053810543.

13 G. Sun, Antimicrobial Textiles: Applications of Antimicrobial Textiles, Woodhead publishing, Duxford, U.K., 2008, p. 265.
14 S. H. Jeong, S. Y. Yeo and S. C. Yi, J. Mater. Sci., 2005, 40, 5407-5411.

15 Z. Cao and Y. Sun, J. Biomed. Mater. Res., Part A, 2008, 85, 99107.

16 G. Sun, T. Y. Chen and S. D. Worley, Polymer, 1996, 37, 37533756.

17 O. Rodríguez-Uicab, F. Avilés, P. I. Gonzalez-Chi, G. CanchéEscamilla and S. Duarte-Aranda, Appl. Surf. Sci., 2016, 385, 379-390.

18 P. G. Tortora and B. J. Collier, Understanding textiles, Prentice-Hall, New Jersey, USA., 1997, pp. 153-164.

19 D. D. Evanoff and G. Chumanov, ChemPhysChem, 2005, 6, 1193-1426.

20 D. Chen, X. Qiao, X. Qiu and J. Chen, J. Mater. Sci., 2009, 44, 1076-1081.

21 A. H. Alshehri, M. Jakubowska, A. Mlożniak, M. Horaczek, D. Rudka, C. Free and J. D. Carey, ACS Appl. Mater. Interfaces, 2012, 4, 7007-7010.

22 G. Doria, J. Conde, B. Veigas, L. Giestas, C. Almeida, M. Assuncăo, J. Rosa and P. V. Baptista, Sensors, 2012, 12, 1657-1687.

23 W. Qiang, H. Li and D. Xu, Anal. Methods, 2013, 5, 629-635.

24 K. Rajan, I. Roppolo, A. Chiappone, S. Bocchini, D. Perrone and A. Chiolerio, Nanotechnol., Sci. Appl., 2016, 9, 1-13.

25 M. Jouni, A. Boudenne, G. Boiteux, V. Massardier, B. Garnier and A. Serghei, Polym. Compos., 2013, 34, 778-786.

26 O. Choi and Z. Hu, Environ. Sci. Technol., 2008, 42, 45834588.

27 J. R. Morones, J. L. Elechiguerra, A. Camacho, K. Holt and M. J. Yacaman, Nanotechnology, 2005, 16, 2346-2353.

28 N. Sachinvala, D. V. Parikh, P. Sawhney, S. Chang, J. Mirzawa, W. Jarrett and B. Joiner, Polym. Adv. Technol., 2007, 18, 620-628.

29 J. Ravelin, Sci. Nat., 1869, 11, 93-102.

30 J. Y. Kim, T. Y. Kim and J. Y. Yoon, J. Korean Ind. Eng. Chem., 2009, 20, 251-257.

31 K. K. Goli, N. Gera, X. Liu, B. M. Rao, O. J. Rojas and J. Genzer, ACS Appl. Mater. Interfaces, 2013, 5, 5298-5306.

32 A. Reznickova, Z. Novotna, Z. Kolska and V. Svorcik, Nanoscale Res. Lett., 2014, 9, 305.

33 P. Krystosiak, W. Tomaszewski and E. Megiel, J. Colloid Interface Sci., 2007, 498, 9-21.

34 C. Kang, S. Kim, S. Kim, J. Lee, J. Lee, C. Roh and J. Lee, Carbohydr. Polym., 2016, 151, 1012-1018.

35 C. Marambio-Jones and E. M. V. Hoek, J. Nano Res., 2010, 12, 1531-1551.

36 K. Chaloupka, Y. Malam and A. M. Seifalian, Trends Biotechnol., 2010, 28, 580-588.

37 V. K. Sharma, R. A. Yngard and Y. Lin, Adv. Colloid Interface Sci., 2009, 145, 83-96.

38 S. Ashraf, N. Akhtar, M. A. Ghauri, M. I. Rajoka, Z. M. Khalid and I. Hussain, Nanoscale Res. Lett., 2012, 7, 267.

39 S. Pal, Y. K. Tak and J. M. Song, Appl. Environ. Microbiol., 2007, 73, 1712-1720.

40 I. Sondi and B. Salopek-Sondi, J. Colloid Interface Sci., 2004, 275, 177-182. 
41 M. Balouiri, M. Sadiki and S. K. Ibnsouda, J. Pharm. Anal., 2016, 6, 71-79.

42 Y. Xue, H. Xiao and Y. Zhang, Int. J. Mol. Sci., 2015, 16, 36263655.

43 E. J. Corey, F. Xu and M. C. Noe, J. Am. Chem. Soc., 1997, 119, 12414-12415.

44 Z. Jia, D. Shen and W. Xu, Carbohydr. Res., 2001, 333, 1-6.

45 S. S. Kim, J. E. Park and J. Lee, J. Appl. Polym. Sci., 2011, 199, 2261-2267.
46 K. F. El-tahlawy, M. A. El-bendary, A. G. Elhendawy and S. M. Hudson, Carbohydr. Polym., 2005, 60, 421-430.

47 B. Martel, M. Weltrowski, D. Ruffin and M. Morcellet, J. Appl. Polym. Sci., 2002, 83, 1449-1456.

48 A. P. Gomes, J. F. Mano, J. A. Queiroz and I. C. Gouveia, Polym. Adv. Technol., 2006, 79A, 665-674.

49 M. H. Jang, S. J. Bae, S. K. Lee, Y. J. Lee and Y. S. Hwang, J. Nanosci. Nanotechnol., 2014, 14, 9665-9669.

50 D. C. Tien, C. Y. Liao, J. C. Huang, K. H. Tseung and J. K. Lung, Rev. Adv. Mater. Sci., 2008, 18, 750-756. 